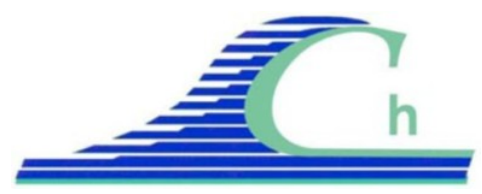

XII ${ }^{\text {èmes }}$ Journées Nationales Génie Côtier - Génie Civil

Cherbourg, 12-14 juin 2012

DOI:10.5150/jngcgc.2012.064-G C Editions Paralia CFL

disponible en ligne - http://www.paralia.fr - available online

\title{
Monitoring temps réel haute résolution d'un littoral : MAGOBS (Villeneuve-lès-Maguelone, Golfe du Lion)
}

\author{
Bénédicte GUERINEL ${ }^{1,3}$, Frédéric BOUCHETTE ${ }^{2,3}$, Olivier LOBRY ${ }^{2}$, \\ Dominique ASTRUC ${ }^{3}$, Pascal AZERAD ${ }^{3}$, Elena BRAMBILLA ${ }^{2,3}$, \\ Raphael CERTAIN ${ }^{3}$, Philippe LARROUDE ${ }^{3}$, Miguel MANNA ${ }^{3}$, \\ Samuel MEULE ${ }^{3}$, Vincent REY ${ }^{3}$, Nicolas ROBIN ${ }^{3}$, François SABATIER ${ }^{3}$, \\ Damien SOUS $^{3}$, David MARTINIE ${ }^{2}$, Nicolas ARNAUD ${ }^{2}$
}

1. Direction Régionale de l'Environnement, de l'Aménagement et du Logement de Languedoc-Roussillon - 520 allée Henry II de Montmorency, 34064 Montpellier Cedex, France. benedicte.guerinel@developpement-durable.gouv.fr

2. OSU OREME ou Géosciences-M CNRS / Université de Montpellier II, France. frederic.bouchette@soltc.org

3. GLADYS / SO LTC groupe Littoral d'Etude de la Dynamique Sédimentaire, regroupant des personnels de 8 laboratoires dans le Grand Sud de la France.

\section{Résumé :}

Le lido de Villeneuve-lès-Maguelone (Languedoc-Roussillon) est un archétype de littoral microtidal, dominé par la houle, en érosion, et largement affecté par la submersion marine lors des tempêtes. Les observations réalisées dans le cadre du Système d'Observation Littoral - Trait de Côte (SO LTC) concernent l'hydrodynamique et le transport sédimentaire dans l'avant-côte et la lagune. La tache d'observation MAGOBS (Observatoire Aresquiers-Maguelone-Palavas) de cet observatoire regroupe un dispositif instrumental automatisé composé de 30 points d'acquisition en temps réel entre 6 mètres de profondeur et le haut de plage (à terre) ainsi que dans la lagune. Le dispositif est opérationnel depuis mai 2011. Son déploiement a permis de déverrouiller les difficultés techniques liées à la mesure temps réel en zone littorale : choix du type des appareils de mesure, de la technologie embarquée, des cadences d'acquisition, résolution des questions du transfert de la donnée. Le dispositif produit 2 Tera-octets de données par an. Les données sont stockées sur un ensemble de serveurs dédiés. Les données sont mises en ligne sur le site du SO-LTC (www.soltc.org), immédiatement après premiers traitements et qualification. Elles commencent à être utilisées pour des calculs de niveau d'eau à la côte, et dans des outils de prédiction des vagues et des niveaux à la côte associant mesure et modélisation.

\section{Mots-clés :}

Instrumentation - Hydrographie - Risques côtiers - Système d'alerte - Submersion Vague - Courantologie - Niveau d'eau - Etat de mer 


\section{Introduction}

La mesure en zone strictement littorale -dans la zone de transformation des vagues, dans la zone de swash et sur la plage émergée- est fondamentale pour la recherche académique comme pour l'ingénierie et l'aménagement littoral. Mesurer les vagues, le niveau d'eau et les courants à la côte apporte des informations objectives à confronter aux restitutions des modélisations numériques littorales. Les modèles actuels calculent de manière approchée et sur de petits périmètres l'hydrodynamique dans les très faibles bathymétries à partir de données acquises au large sur le plateau continental, par 20 à 30 mètres de fonds. Ainsi, le principe d'un système de mesure long terme, temps réel et haute résolution des processus hydro-sédimentaires le long du trait de côte (figure 1) constitue une avancée notable en terme d'intérêt scientifique et d'ingénierie, mais également de développement technologique.

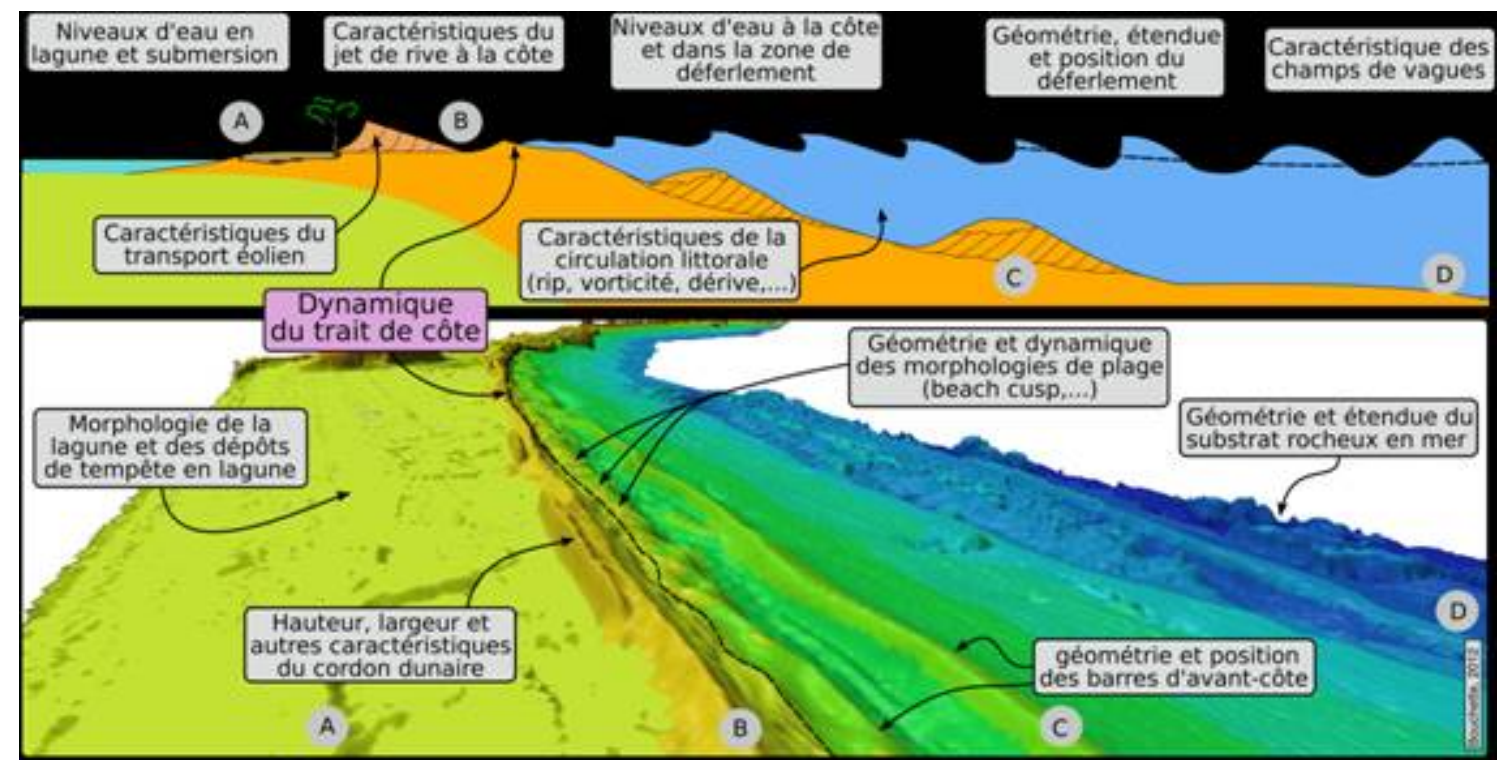

Figure 1. les phénomènes physiques hydrodynamiques (en haut, 1.) et les morphologies (en bas, 2.) affectant l'arrière dune (A), la plage émergée et le cordon dunaire (B), la zone de déferlement et les barres d'avant-côte $(C)$, et la zone de transformation des vagues $(D)$.

Tous ces processus se combinent et participent à la dynamique du trait de côte.

De nombreuses campagnes de mesure hydrodynamique ont été menées depuis les années 80 en France, sur des périodes allant de quelques jours à plusieurs mois, les plus longues étant réalisées en général dans le cadre de grands chantiers d'aménagement du littoral. Cet article présente MAGOBS, le premier système de suivi hydro-sédimentaire strictement littoral déployé sur la façade méditerranéenne. 


\section{XII ${ }^{\text {èmes }}$ Journées Nationales Génie Côtier - Génie Civil \\ Cherbourg, 12-14 juin 2012}

\section{L'observatoire de Maguelone : MAGOBS}

\subsection{Le dispositif technique}

Le dispositif MAGOBS a été déployé dans la partie Ouest du Golfe d'Aigues-Mortes, entre la ville de Frontignan et la ville de Palavas-les-Flots, au niveau de Villeneuve-lèsMaguelone (figure 2), sur un segment littoral dont on trouvera une description morphologique dans CAMPMAS et al. (2012) et dont les caractéristiques sédimentaires sont parfaitement connues (RAYNAL et al., 2010).

La stratégie d'instrumentation du site est de viser l'exhaustivité technologique en matière de mesure, en mettant en œuvre des dispositifs reconnus dans leur domaine. Ainsi, le dispositif est composé d'un ensemble d'appareils permettant la mesure de la courantologie le long de profils horizontaux ou verticaux (ADCP NORTEK, AQUAPRO NORTEK) ou ponctuellement (ADV NORTEK), la mesure de la période et de la hauteur des vagues (mesure de la forme de la surface via effet doppler), des niveaux d'eau (pressiomètres) et de la charge sédimentaire en suspension dans la colonne d'eau (turbidimètre OBS), ainsi que la mesure de paramètres plus généraux (vent, température air/eau, salinité, ...). L'ensemble des équipements est intégré en réseau via un système de câbles lestés par des chaînes de maillons de $12 \mathrm{~cm}$ à $30 \mathrm{~cm}$ de longueur selon l'emplacement et l'exposition aux vagues.

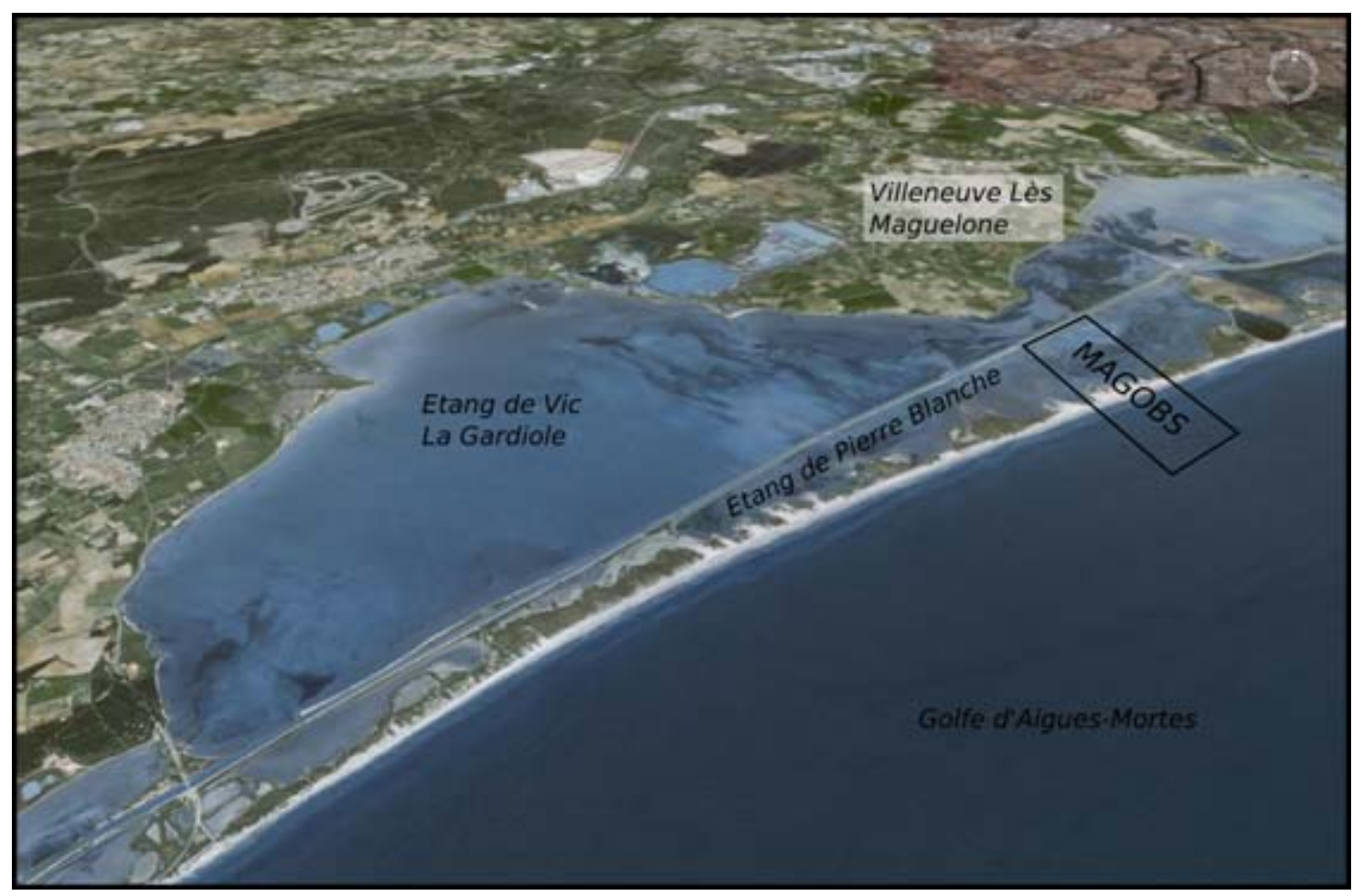

Figure 2. Emplacement du dispositif MAGOBS, sur le lido de Villeneuve-lès-

Maguelone. La zone est notamment caractérisée par sa forte exposition au processus de submersion. 
Les équipements eux-mêmes sont ancrés sur le fond selon plusieurs méthodes (vis tournante et poteaux en lagunes; tubes creux en $150 \mathrm{~mm}$ sur $6 \mathrm{~m}$ de long, double dalle béton $4 \mathrm{~m}^{2}$ et barnacle en mer ; tubes creux sur le lido sableux).

La totalité de l'information passe par ce réseau de câbles disposés en étoile autour d'un petit local posé en hauteur sur la plage et appelé BJP (Boitier Jonction Principal). Le rôle du BJP est de regrouper les données, répartir les commandes, alimenter les appareils. Le BJP est lui-même connecté à un Central Acquisition (CA) déporté à 1 km en dehors de la zone directement exposée aux vagues et à la submersion marine. La connexion est faite via un ombilical enterré dans une tranchée protégée; le signal entre BJP et CA est modulé pour être transporté sur de grandes distances si nécessaire. Au niveau du CA, un système informatique stocke l'ensemble des mesures acquises, réalise des mesures complémentaires (base GPS, vent et autres mesures météorologiques) et dispose d'un système pour assurer le transfert temps réel (via GPRS, internet) des données choisies vers les serveurs de Bases de Données de l'OSU OREME. A plein régime, ce dispositif représente un flux annuel de 2 To de données entre les appareils déployés en mer et les bases stockées à l'OSU OREME.

\subsection{Les mesures réalisées entre mai 2011 et mai 2012}

Sur la première année de fonctionnement, le dispositif MAGOBS se présente tel que décrit sur la figure 3.

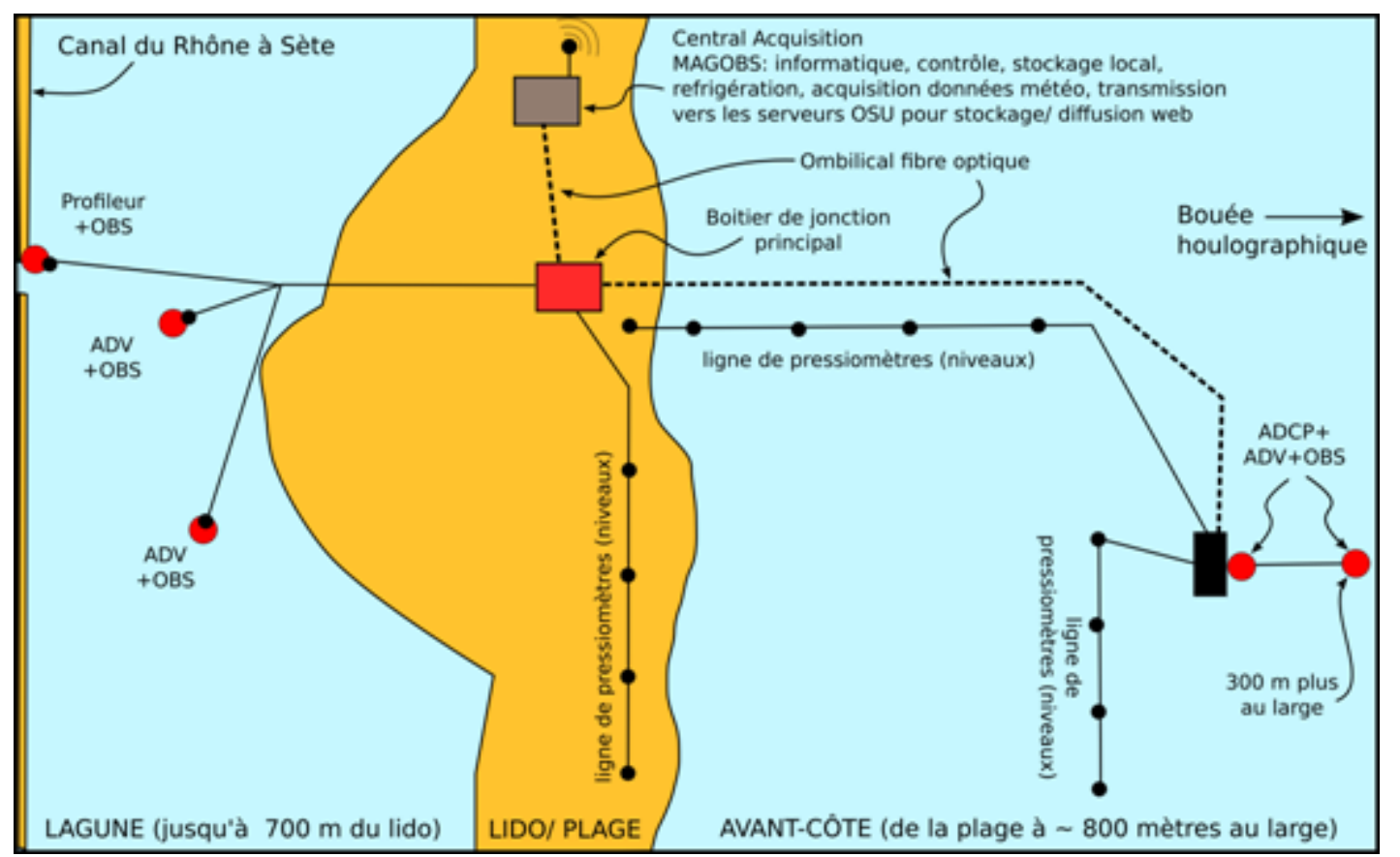

Figure 3. Le dispositif MAGOBS sur la période mai 2011/ mai 2012. Les cercles rouges sont des points où sont disposés plusieurs équipements. Les cercles noirs sont des points de mesure du niveau marin. Les distances horizontales ne sont pas respectées. 


\section{XII ${ }^{\text {èmes }}$ Journées Nationales Génie Côtier - Génie Civil \\ Cherbourg, 12-14 juin 2012}

Les mesures réalisées sur le dispositif MAGOBS sont les suivantes :

1) les caractéristiques des vagues (période et hauteur) en 3 points de mesure dans la lagune, et 2 points de mesure sur l'avant-côte ;

2) les niveaux d'eau en 17 points dont 5 sur le lido sableux (uniquement actif durant les submersions) ;

3) la courantologie en 5 points de mesure dont 2 en mer et 3 en lagune ;

4) une mesure de vent à 7 mètres au niveau du CA ;

5) une mesure de station de base GPS au niveau du CA ;

6) des mesures de température de l'eau en 5 points ( 2 en mer, 3 en lagune).

Le dispositif MAGOBS ne repose pas sur une transmission ininterrompue de données (burst). En effet, l'ensemble des équipements réalise des mesures à $2 \mathrm{~Hz}$ en continu, sauf les mesures de houle qui sont réalisées à $4 \mathrm{~Hz}$ également en continu. Les données brutes sont stockées sur le serveur du CA. Toutes les 10 minutes, des moyennes et autres traitements de base sont réalisés sur les données brutes et les valeurs calculées sont transmises via internet sur les bases de données à l'OSU OREME. Les données brutes sont rapatriées sur les bases de données indépendamment, et hors flux temps réel.

\subsection{L'interface de consultation / intégration dans un système d'observation}

Selon les principes des OSU, l'OREME et la DREAL LR se sont regroupés pour développer un système de stockage/sécurisation/diffusion de l'ensemble des données issues des campagnes de mesure hydro-morphodynamique sur la façade Méditerranéenne, ainsi que des données historiques (cartes), morphologiques (profils altimétriques et bathymétriques, LIDAR, photo aériennes, ...), et sédimentologique/ géologique/géochimique (sismique, carottages, mesure de proxies). Cette collection d'informations est organisée dans un ensemble de bases de données appelé le Système d'Observation Littoral - Trait de Côte (SO LTC), les données stockées ayant toutes un point commun : elles concernent des processus contrôlant la dynamique du trait de côte. Le dispositif MAGOBS est intégré à ce système d'observation, et les données mesurées sont strictement mises en ligne au travers du SO LTC (www.soltc.org). Plus précisément, les données MAGOBS sont :

- soit accessibles en temps que telles, au travers d'un outil de consultation de la base de données propre à MAGOBS ;

- soit accessibles via des produits web dérivés, où les données de MAGOBS sont associées à d'autres données (profil topo, données issues de bouées houlographique, ...) pour en augmenter l'intérêt, et permettre aux utilisateurs de croiser plusieurs types d'information.

La figure 4 montre une copie d'écran d'une des pages de l'outil de consultation de MAGOBS. 


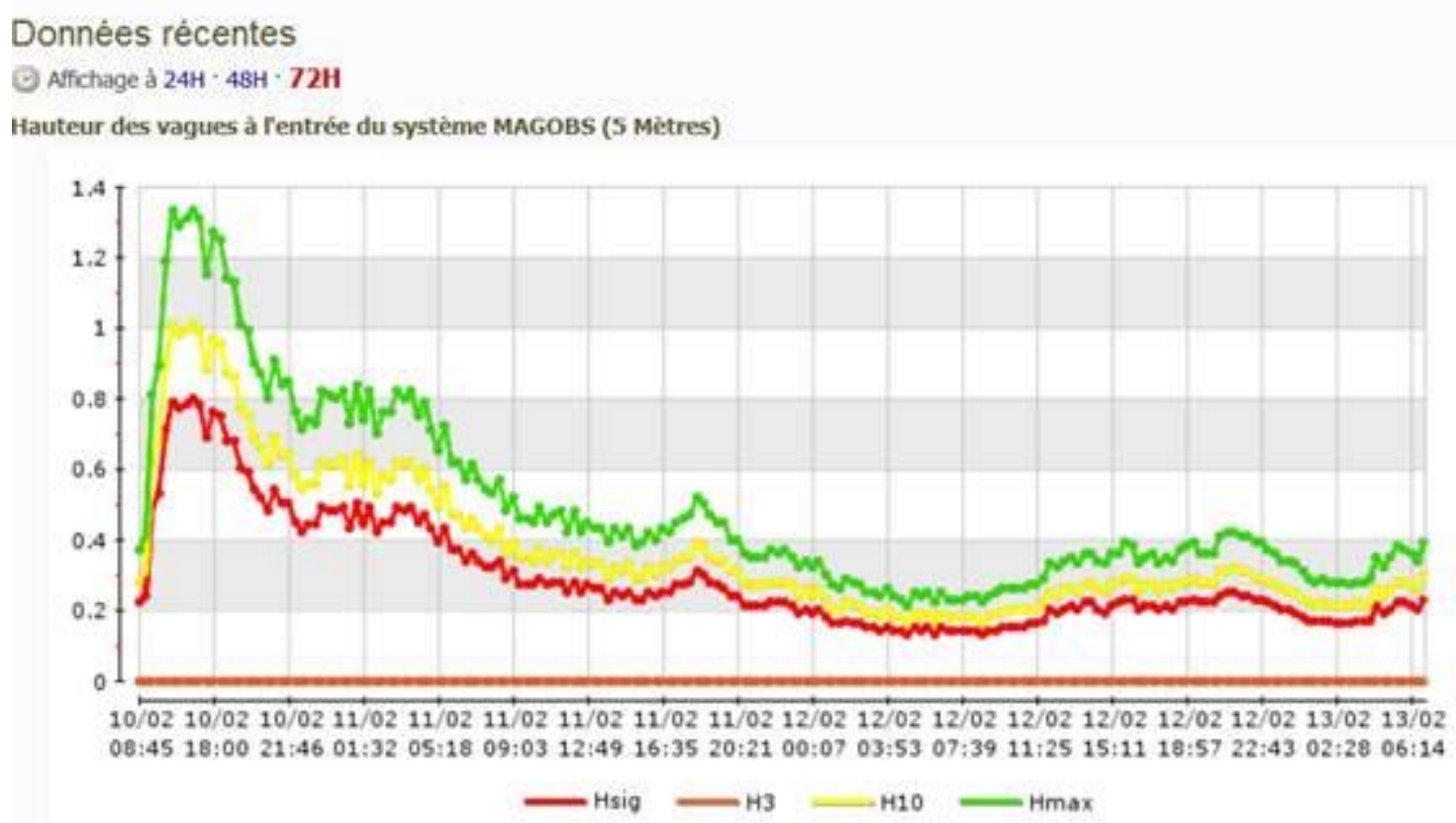

Figure 4. Un extrait de l'outil de consultation web des données issues de MAGOBS, au travers du système d'observation Littoral - Trait de Côte.

\section{L'utilisation des données MAGOBS dans le SO LTC}

Les données MAGOBS vont $a b$ minima :

1) permettre d'acquérir de la connaissance sur le fonctionnement du littoral ;

2) servir de point de mesure supplémentaire des conditions météo-marines dans le Golfe du Lion, au même titre que les bouées houlographiques de Sète, Espiguette, Leucate et Banyuls ;

3) servir de données de forçage/validation d'un outil de calcul de la submersion du lido par les vagues (BOUCHETTE et al., 2012) ;

4) servir à alimenter un outil d'alerte à la submersion, puisque le système délivre toute les 10 minutes un état du niveau marin précis sur un segment littoral ;

5) servir de point de calibration/validation pour les modèles numériques portant sur l'hydrodynamique du proche plateau continental et du littoral du Golfe du Lion (e.g. LEREDDE et al., 2007).

\section{Un exemple d'utilisation opérationnelle des données MAGOBS}

Entre le 23 octobre et le 06 novembre 2011, le Languedoc Roussillon a subi un épisode météorologique marquant avec plusieurs épisodes cévenols. Au cours de ces événements a été enregistrée une baisse de la pression atmosphérique ramenée au niveau de la mer, accompagnée de vents très forts et de vagues de secteur Est à Sud Est. Le dispositif MAGOBS a permis de suivre spécifiquement les forçages météorologiques et hydrodynamiques sur le site et de les comparer à ceux enregistrés au large. La 


\section{XII ${ }^{\text {èmes }}$ Journées Nationales Génie Côtier - Génie Civil \\ Cherbourg, 12-14 juin 2012}

comparaison des données montre une bonne corrélation entre les sites au large et le site MAGOBS mais semble également mettre en évidence des effets de site, à analyser plus précisément sur plus long terme.
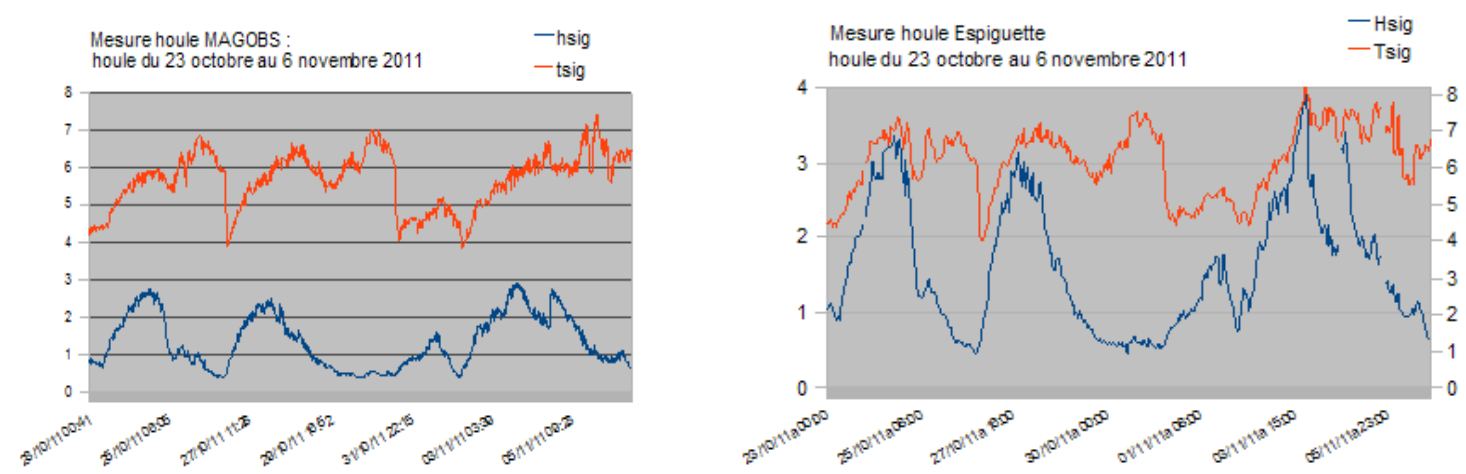

Figure 5. Comparaison de forçages mesurés à la bouée de l'Espiguette et au droit du dispositif MAGOBS.

\section{Conclusion}

Le système MAGOBS est la première initiative académique française de suivi temps réel de l'hydrodynamique strictement littorale (au trait de côte) haute résolution spatiale et haute fréquence temporelle.

La première année est très positive pour toutes les mesures en relation avec la circulation et les caractéristiques des vagues, ainsi que les mesures de niveau d'eau par des pressiomètres embarqués. On observe quelques limitations dans l'utilisation des pressiomètres isolés, dont le fonctionnement semble être bloqué par l'ensablement.

$\mathrm{Au}$ niveau de la transmission des données, la solution filaire retenue fonctionne parfaitement, et les systèmes d'ancrage sur le fond sont également très efficaces. Des clones améliorés du dispositif MAGOBS, appelés LEUCATOBS et ESPIGOBS ont vocation à être déployés respectivement au-devant de la plage de Leucate et au droit du phare de l'Espiguette. LEUCATOBS sera orienté zone de déferlement. ESPIGOBS sera axé sur la question de l'hydrodynamique sur une flèche sableuse et la dérive littorale. Ces systèmes instrumentés ont vocation à intégrer le dispositif BIGBLUE, généralisation du système MAGOBS à l'ensemble du bassin Méditerranéen, avec un stockage centralisé au niveau du Système d'Observation LTC.

Au niveau des développements logiciels, MAGOBS est en cours d'intégration dans l'atlas hydrodynamique du littoral (www.atlashydrolittoral.org). MAGOBS est également progressivement intégré dans des outils d'alerte à la submersion, combinant mesure et modèle. L'état d'avancement des travaux est précisé sur le site www.soltc.org. 
Thème 3 - Instrumentation, mesures, imagerie et télédétection

\section{Remerciements :}

Nos remerciements vont à l'ensemble des tutelles nationales (ministères, INSU) et régionales (Université Montpellier II, DREAL LR, Région Languedoc-Roussillon) permettant le développement de ces dispositifs innovants. Nous remercions également l'alliance ALLENVI pour la labellisation SOERE du dispositif MAGOBS, dans le cadre du SOERE Trait de côte. Nous remercions la Région Languedoc-Roussillon pour son soutien apporté au grand plateau technique (GEPETO) GLADYS depuis 2005 et pour le renouvellement récent de sa confiance au travers du prix Chercheur d'Avenir 2012.

\section{Références}

BOUCHETTE F., SABATIER F., SYLAIOS G., MEULE S., LIOU JY, HEURTEFEUX H., DENAMIEL C., HWUNG H.H. (2012). SUBDUNE tool: quasiexplicit formulation of the water level along the shoreline. XII ${ }^{\text {èmes }}$ Journées Nationales Génie Côtier- Génie Civil, Cherbourg. doi:10.5150/jngcgc.2012.022-B

CAMPMAS L, BOUCHETTE F., LIOU JY, BRAMBILLA E, MEULE S., SYLAIOS G., SABATIER F., CERTAIN R., ROBIN N., HWUNG H.H. (2012). Upper beach reconstruction patterns after moderate storms events XII ${ }^{\text {èmes }}$ Journées Nationales Génie Côtier- Génie Civil, Cherbourg. doi:10.5150/jngcgc.2012.023-C

LEREDDE Y., DENAMIEL C., BRAMBILLA E., LAUER-LEREDDE C., BOUCHETTE F., MARSALEIX P. (2007). Hydrodynamics in the Gulf of AiguesMortes, NW Mediterranean Sea: in-situ data and modelling. Continental Shelf Research. Volume 27 (18), pp 2389-2406. doi:10.1016/j.csr.2007.06.006

RAYNAL O., BOUCHETTE F., CERTAIN R., SABATIER P., LOFI J., SERANNE M., DEZILEAU L., BRIQUEU L., FERRER P., COURP T. (2010). Holocene evolution of a Languedocian lagoonal environment controlled by inherited coastal morphology (northern Gulf of Lions, France). Bulletin de la Société géologique de France. Vol. 181 (2), pp 211-224. doi: 10.2113/gssgfbull.181.2.211 